\title{
Bioatividade da Cabralea canjerana (Vell.) Mart. (Meliaceae) no controle de adultos de Acanthoscelides obtectus (Coleoptera, Bruchidae) em laboratório
}

\author{
Lisonéia Smaniotto ${ }^{1}$ \\ Neusa Fernandes de Moura ${ }^{1 *}$ \\ Rosiane B. N. Denardin ${ }^{1}$ \\ Flávio R. M. Garcia ${ }^{2}$ \\ 'Área de Ciências Exatas e Ambientais - UNOCHAPECÓ \\ Caixa Postal 1141, CEP 89809-000, Chapecó - SC, Brasil \\ ${ }^{2}$ Instituto de Biologia, Departamento de Zoologia e Genética \\ Universidade Federal de Pelotas, Pelotas - RS, Brasil \\ *Autor para correspondência \\ nfm@unochapeco.edu.br
}

\section{Resumo}

A bioatividade de Cabralea canjerana (Vell.) Mart., espécie arbórea, comum na região Oeste de Santa Catarina, foi testada frente ao Acanthoscelides obtectus Say (Coleptera: Bruchidae), uma importante praga que danifica o grão do feijão. No experimento foram utilizados 10 insetos adultos, colocados em placas de Petry revestidas de papel filtro, onde foram adicionadas alíquotas de extrato bruto, fração hexânica, clorofórmica, acetato de etila e óleo essencial de folhas de C. canjerana nas concentração de $10 \%, 5 \%$ e $1 \%$. O resultado em laboratório demonstrou eficiência de $100 \%$ para o extrato bruto (concentração $1 \%$ ), seguido da fração hexânica (concentração 1\%) com eficiência de 84,2\%. As frações acetato de etila, clorofórmica e óleo essencial foram as que apresentaram as menores eficiências no controle do Acanthoscelides obtectus.

Unitermos: caruncho do feijão, controle de pragas, plantas inseticidas

\section{Abstract}

Bioactivity of Cabralea canjerana (Vell.) Mart. (Meliaceae) in the control of Acanthoscelides obtectus (Coleoptera, Bruchidae) in the laboratory. The bioactivity of Cabralea canjerana (Vell.) Mart, a common tree species in the western region of Santa Catarina, was tested with Acanthoscelides obtectus Say (Coleptera: Bruchidae), an important insect-pest that can cause damage to bean seeds. In the experiment, 10 adult insects were put onto Petry dishes lined with filter paper, to wich where was add aliquots of crude extract, hexanic, chloroformic, ethyl acetate fractions and essential oil of Cabralea canjerana leaves, concentrated in 10\%, 5\% and $1 \%$, were added. The results in the laboratory showed $100 \%$ efficiency of the crude extract (concentration $1 \%$ ), followed by the hexanic fraction (concentration 1\%) with $84.2 \%$. The ethyl acetate, chloroformic fractions and essential oil showed lower efficiency in the control of Acanthoscelides obtectus.

Key words: bean insect-pest, insecticidal plants, pest control 


\section{Introdução}

O feijão (Phaseolus vulgaris) é uma das culturas de maior importância para a alimentação humana, consiste em uma excelente fonte protéica, apresentando bom conteúdo de carboidratos, rico em ferro, e, além disso, apresenta grande importância econômica no Brasil, sendo fonte de renda para muitos agricultores, muito deles de agricultura familiar. Porém, o cultivo do feijão apresenta vários problemas, entre eles destaca-se o caruncho do feijão (Asconthoscelides obtectus), que afeta tanto na fase de lavoura como no seu armazenamento. Este inseto, além de atacar os diversos estágios de desenvolvimento, danifica os grãos quando estão em fase de armazenamento, onde abre galeria nos grãos, o que provoca a destruição completa do grão, impossibilitando a sua comercialização tanto para alimentação como propagação (Gallo, 2002).

Atualmente tem aumentado o interesse em pesquisas que utilizam extratos de plantas como alternativa aos inseticidas sintéticos, principalmente pela necessidade de dispor de novos compostos para controle de pragas, já que inseticidas naturais são obtidos de recursos renováveis e rapidamente são degradados, não deixando resíduos em alimentos.

Cabralea canjerana (Velloso) Martius é uma das espécies madeireiras mais valiosas do sul do Brasil. Conhecida popularmente por canjarana ou canjerana, a sua ocorrência vai desde Minas Gerais, Mato Grosso do Sul até o Rio Grande do Sul, principalmente em mata pluvial da encosta atlântica (Lorenzi, 2000). Pertencente a família das Meliaceae que é bastante conhecida pelo seu potencial inseticida (Costa et al., 2004), a espécie Cabralea canjerana também apresenta compostos químicos com este potencial, decorrentes principalmente pela presença de triterpenos do tipo damarano e limonóides (Soares et al., 2004).

O objetivo deste trabalho é avaliar a bioatividade de Cabralea canjerana e sua eficiência no controle do caruncho do feijão (Acanthoscelides obtectus) em laboratório, praga esta, que pode provocar a destruição completa do feijão, levando a sua depreciação comercial.

\section{Material e Métodos}

\section{Coleta do material}

O material botânico foi coletado no município de Chapecó, SC, identificada pelo Herbário da UFSM, e sua exsicata depositada sob número SMDB 12.356 no mesmo Herbário. Os espécimes de A. obtectus foram coletados no mesmo município, identificado e acondicionados no Laboratório de Entomologia da UNOCHAPECÓ.

\section{Obtenção dos óleos}

A espécie vegetal foi coletada na primavera, sendo que suas folhas "in natura", foram secas à sombra, posteriormente maceradas e submetidas à extração através de arraste de vapor por quatro horas utilizando aparelho de "Clevenger" modificado. A separação do óleo com água foi realizada utilizando éter etílico, secando a fase orgânica com sulfato de sódio anidro e evaporando o solvente em rotaevaporador (evaporador rotativo Rampolph).

\section{Obtenção do extrato e frações}

As folhas da espécie Cabralea canjerana foram secas a sombra, após foi realizado a maceração em álcool etílico por 48 horas e, posteriormente, filtradas em papel filtro. A solução foi concentrada em evaporador rotativo Ramdolph para a eliminação do álcool, produzindo assim extrato bruto (EB). O EB sofreu fracionamento liquidoliquido com solventes orgânicos, em ordem crescente de polaridade, resultando em fração hexânica $(\mathrm{FH})$, fração clorofórmica (FC) e fração acetato de etila (FA). Para a retirada dos solventes, os extratos e frações passaram no evaporador rotativo Ramdolph. Após a retirada do solvente, os extratos e frações foram liofilizados para a retirada de água e dos possíveis resíduos do solvente.

\section{Aplicação em laboratório}

$\mathrm{O}$ experimento foi realizado, sob temperatura de $25^{\circ} \mathrm{C} \pm 3{ }^{\circ} \mathrm{C}$ e umidade relativa do ar de $70 \% \pm 10 \%$, com fotoperíodo de $12 \mathrm{~h}$. 
Para o ensaio foram utilizadas placas de Petry com $10 \mathrm{~cm}$ de diâmetro e $2 \mathrm{~cm}$ de altura, o interior das placas foi coberto com papel filtro, onde foram adicionadas alíquotas do óleo e extratos em concentrações de $1 \%$, $5 \%$ e $10 \%$, e como testemunha foi utilizado água destilada. Foram utilizados apenas insetos adultos acondicionados em número de dez insetos por placa (unidade experimental). Para cada tratamento foram realizados quatro repetições em delineamento totalmente casualizado. A contagem de indivíduos vivos (caruncho do feijão) foi realizada a cada doze horas durante três dias. A análise estatística foi realizada utilizando-se o software livre Sisvar e as médias foram agrupadas pelo teste de Tukey com nível de significância a $0,05 \%$. A eficiência dos diferentes tratamentos foi obtida pela aplicação da fórmula de Abott (1925).

\section{Resultado e Discussão}

A avaliação da eficiência da $C$. canjerana sobre adultos de $A$. obtectus foi realizado utilizando o óleo essencial, extrato bruto, fração hexânica, fração clorofórmica e fração acetato de etila da espécie.

Na Tabela 1, observa-se que o extrato bruto (EB) da canjerana apresenta diferença significativa $(\mathrm{P}<0.05)$ entre o tratamento e a testemunha, sendo que o tratamento com $1 \%$ apresentou eficiência de $100 \%$. $\mathrm{O}$ que demonstra que a menor concentração foi a mais eficiente no controle do caruncho.

TABELA 1: Médiasdeadultos vivosdeAcanthoscelides obtecus submetidos à aplicação de Cabralea canjerana.

\begin{tabular}{lcccc}
\hline & $\begin{array}{c}\text { Concen- } \\
\text { tração } \\
\mathbf{1 \%}\end{array}$ & $\begin{array}{c}\text { Concen- } \\
\text { tração } \\
\mathbf{5 \%}\end{array}$ & $\begin{array}{c}\text { Concen- } \\
\text { tração } \\
\mathbf{1 0 \%}\end{array}$ & Controle \\
\hline Extrato bruto & $0 \mathrm{a}$ & $2,2 \mathrm{~b}$ & $2,7 \mathrm{~b}$ & $9,5 \mathrm{c}$ \\
Fração hexânica & $1,5 \mathrm{a}$ & $5,5 \mathrm{c}$ & $3,8 \mathrm{~b}$ & $9,5 \mathrm{c}$ \\
Fração clorofórmica & $5,2 \mathrm{a}$ & $6,0 \mathrm{ab}$ & $7,5 \mathrm{~b}$ & $9,5 \mathrm{c}$ \\
Fração acetato & $6,0 \mathrm{~b}$ & $8,5 \mathrm{c}$ & $4,2 \mathrm{a}$ & $9,5 \mathrm{c}$ \\
Óleo essencial & $7,6 \mathrm{c}$ & $4,8 \mathrm{~b}$ & $3,0 \mathrm{a}$ & $9,5 \mathrm{~d}$ \\
\hline
\end{tabular}

Tratamentos seguidos da mesma letra em horizontal não diferem estatisticamente $(\mathrm{P}<0.05)$.

Diferentemente, sobre outras pragas como a Brevycorine brassicae (L.) (Hemiptera, Aphididae) e Ascia monuste orseis (Lepidoptera, Pieridae), o extrato bruto da espécie $C$. canjerana apresentou maior eficiência quando utilizada maiores concentrações $(10 \%)$, sendo que menores não apresentaram efeito significativo (Mata, 2007).

A mesma relação pode ser observada nas frações hexânica (FH) e clorofórmica (FC), onde os tratamentos apresentaram diferença significativa entre si, sendo o melhor tratamento para a FH e FC a 1\%. Estas frações apresentam compostos com baixa polaridade, como no caso de diterpenos que segundo Aslan et al. (2006) apresentam atividade frente ao Acanthoscelides obtecus. Pemonge et al. (1997) também observaram para a fração hexânica de Trigonella foenun considerável ação inseticida sobre o Acanthoscelides obtecus.

Já para a fração acetato de etila (FA), o tratamento de maior concentração foi o mais eficiente, sendo que com concentração de $5 \%$ não houve diferença significativa $(\mathrm{P}<0.05)$ com a testemunha.

Resultado semelhante foi observado para a Trigonella foenun sobre o Acanthoscelides obtecus, onde a fração acetona não demonstrou efeito sobre o inseto (Pemonge et al., 1997).

No caso da Cabralea canjerana, a maior concentração de compostos com atividade inseticida encontra-se principalmente nas frações de menor polaridade (Braga et al., 2006), consequentemente as frações polares, no caso, a fração acetato de etila, apresente pouca eficiência.

Os óleos essenciais de espécies vegetais apresentam ação inseticida pela presença de compostos terpenóides (Viegas, 2003). Em trabalho de Regnault-Roger et al. (2004) foi observada eficiência dos monoterpenos oxigenados como o carvacrol, linalol, eugenol e timol sobre o Acanthoscelides obtecus.

Para o óleo essencial da Cabralea canjerana sobre o Acanthoscelides obtecus, pode-se observar que todas as concentrações foram efetivas sobre a praga, diferindo significativamente da testemunha e aumentando sua atividade com o aumento da concentração do óleo essencial.

Espécies pertencente a família das Lamiaceae, também demonstraram atividade significativa sobre o 
Acanthoscelides obtectus. Esta atividade foi observada para o óleo essencial da Mentha microphyla, Rosmarinus officinalis e Oreganum vulgaris no controle dos ovos, larva, pulpa e adultos do Acanthoscelides obtecutus (Papachristos e Stamopoulos, 2002a; 2002b; 2004).

Com relação à eficiência do extrato bruto, frações e óleo essencial da Cabralea canjerana, a Tabela 2 apresenta, segundo a fórmula de Abott (1995), o percentual de eficiência sobre o Acanthoscelides obtectus.

TABELA 2: Eficiência dos tratamentos da Cabralea canjerana sobre Acanthoscelides obtecus.

\begin{tabular}{l|c|c|c}
\hline \multicolumn{1}{c}{} & $\begin{array}{c}\text { Concentra- } \\
\text { ção }\end{array}$ & $\begin{array}{c}\text { Concentra- } \\
\text { ção }\end{array}$ & $\begin{array}{c}\text { Concentra- } \\
\text { ção }\end{array}$ \\
& $\mathbf{1 \%}$ & $\mathbf{5 \%}$ & $\mathbf{1 0 \%}$ \\
\hline Extrato bruto & 100,00 & 76,31 & 71,05 \\
Fração hexânica & 84,21 & 42,10 & 60,53 \\
Fração clorofórmica & 36,84 & 44,74 & 21,05 \\
Fração acetato & 36,84 & 10,52 & 55,26 \\
Óleo essencial & 21,05 & 50,00 & 68,42 \\
\hline
\end{tabular}

Observa-se que o EB a 1\% apresenta a melhor eficiência a 100\%. Procópio e Vendramini (1997) também observaram mortalidade de $100 \%$ de Acanthoscelides. obtectus, utilizando pós (material moído) de folhas, flores e frutos Chenopodium ambrosioides.

As frações hexânica (FH) $1 \%$ e extrato bruto (EB) a $5 \%$ apresentaram eficiência superior a $75 \%$. Os demais apresentaram eficácia inferior a $75 \%$, sendo que fração clorofórmica (FC) a 10\%, óleo essencial 1\% e fração acetato (FA) a $5 \%$, a eficiência foi inferior a $25 \%$.

A Figura 1 resume a análise comparativa entre o extrato bruto, frações e óleo essencial da Cabralea canjerana sobre o Acanthoscelides obtecus. Observa-se que o melhor tratamento foi com o extrato bruto, seguido da fração hexânica. As demais frações e óleo essencial não apresentaram diferença significativa entre si.

Este resultado pode ser atribuído ao fato dos compostos de maior atividade, no caso triterpenos presentes na Cabralea canjerana encontram-se em frações apolares (Braga et al., 2006).

No caso do óleo essencial da espécie Cabralea canjerana, não existe até o momento estudos da sua composição química, porém sabe-se que monoterpenos apresentam ação tóxica sobre $\mathrm{o}$ A. obtecus (Papachristos e Stamopoulos, 2002a; 2004). Desta forma, acreditase que a atividade observada para o óleo da Cabralea canjerana deve-se a presença destes compostos.

\section{Referências}

Abott, W. S. 1925. A method for computing the effectiveness of an insecticide. Journal of Economical Entomology, 18: 265-267.

Aslan, I.; Kilic, T.; Goren, A. C.; Gulacti, T. 2006. Toxicity of acetone extract of Sideritis trojana and 7-epicandicandiol, 7-epicandicandiol diacetate and 18-acetylsideroxol against stored pests Acanthoscelides obtectus (Say), Sitophilus granarius (L.) and Ephestia kuehniella (Zell.). Industrial Crops and Products, 23: 171-176.

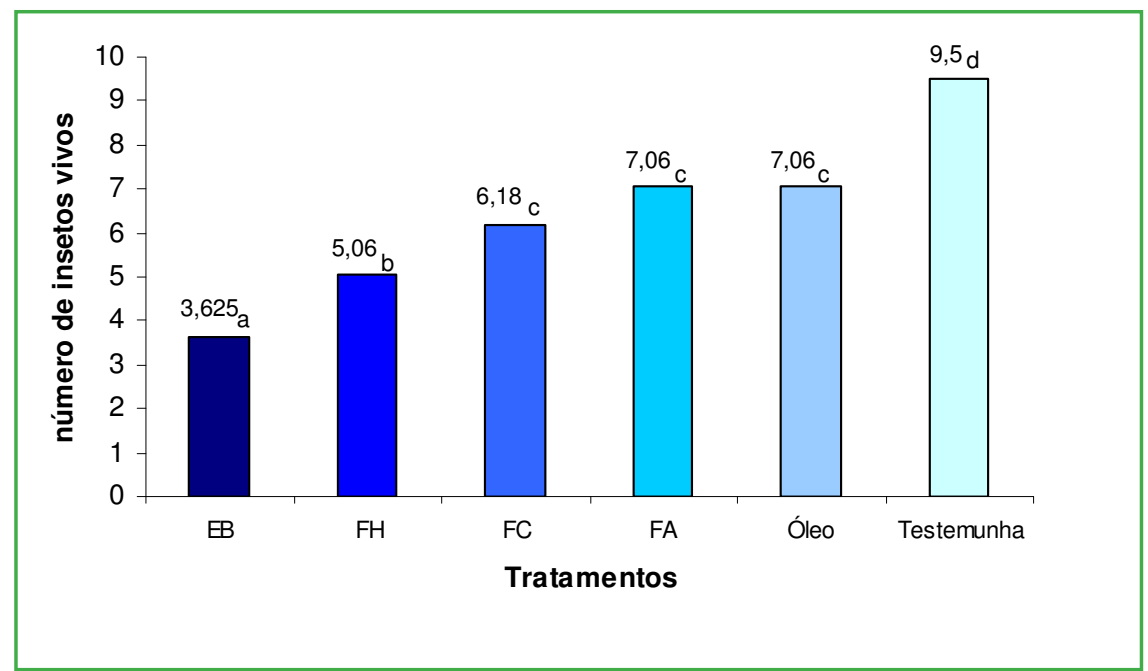

FIGURA 1: Médias de insetos vivos de Acanthoscelides obtecus submetidos à aplicação do extrato bruto, fracionados e óleo essencial de Cabralea canjerana (médias seguidas da mesma letra não diferem, $\mathrm{P}<0,05$ ). 
Braga, P. A. C.; Soares, M. S.; Silva, M. F. G. F.; Vieira, P. C.; Fernandes, J. B.; Pinheiro, A. L. 2006. Dammarane triterpene from Cabralea canjerana (Vell) Mart. (Meliaceae): Theyr chemosystematic significance. Biochemical Systematic and Ecology, 34: 282-290.

Costa, E. L. N.; Silva, R. F. O.; Fiuza, L. M. 2004. Efeitos, aplicações e limitações de extrato de plantas inseticidas. Acta Biológica Leopoldinense, 26 (2): 173-185.

Gallo, D. 2002. Entomologia agrícola. 10ª ed. FEALQ, Piracicaba, Brasil, 920pp.

Lorenzi, H. 2000. Árvores brasileiras: Manual de identificação e cultivo de plantas arbóreas nativas do Brasil. $3^{\text {a }}$ ed. Ed. Plantarum, Nova Odessa, Brasil, 355pp.

Mata, R. F. F. R. 2007. Efeito do extrato aquoso de Cabralea canjerana subsp. polytrica (Adr.Juss) Penn. (Meliacea) no controle biológico de Brevycorine brassicae (L.) (Hemiptera: Haphididae) e Ascia monuste orseis (Godart) (Lepitoptera: Pieridae). Dissertação de Mestrado, Universidade Federal de Uberlândia, Brasil, 76pp.

Papachristos, D. P.; Stamoupolous, D. C. 2002a. Repellent, toxic and reproduction inhibitoryeffects of essential oil vapours on Acanthoscelides obtectus (Say) (Coleoptera: Bruchidae). Journal of Stored Product Research, 31:117-128.

Papachristos, D. P.; Stamoupolous, D. C. 2002b. Toxicity of vapours of three essential oils to the immature stages of Acanthoscelides obtectus (Say) (Coleoptera: Bruchidae). Journal of Stored Product Research, 38: 365-373.
Papachristos, D. P.; Stamoupolous, D. C. 2004. Fumigation toxicity of three essential oil on the eggs of Acanthoscelides obtectus (Say) (Coleoptera: Bruchidae). Journal of Stored Product Research, 40: 517-525.

Pemonge, J.; Pascula-Villalobos, M. J.; Regnault-Roger, C. 1997. Effects of material and extracts of Trigonella foenum-graecum L. against the stored product pests Tribolium castaneum (Herbst) (Coleoptera: Tenebrionidae) and Acanthoscelides obtecus (Say) (Coleoptera: Bruchidae). Journal of Stored Product Research, 33 (3): 209-217.

Procopio, S. O.; Vendramim, J. D. 1997. Avaliação da atividade inseticida de diversos pós de origem vegetal em relação a $A$. obtectus (SAY). Anais do XVI Congresso Brasileiro de Entomologia, Salvador, Brasil, p-326.

Regnault-Roger, C.; Ribodeau, M.; Hamraoui, A.; Bareau, I.; Blanchard, P.; Gilmunoz, M. I.; Barberan, F. T. 2004. Polyphenolic compounds of mediterranean Lamiaceae and investigation of orientational effects on Acanthoscelides obtectus (Say). Journal of Stored Product Research, 40: 395-408.

Soares, M. S.; Silva, M. F.das G. F.; Fernandes, J. B.; Vieira, P. C. 2004. Triterpeno e Limonóides isolados dos frutos de Cabralea canjerana. Anais XXVI Reunião Anual sobre Evolução, Sistemática e Ecologia Micromoleculares, Rio de Janeiro, Brasil, p.23-24.

Viegas, C. J. 2003. Terpenos com atividade inseticida: Uma alternativa para o controle químico de insetos. Química Nova, 23 (3): 390-400. 